\title{
Survey of Prevalence and Risk Factors Associated with Upper Extremity Musculoskeletal Disorders by Repetitive Job Activities Methods in Baker of Iran
}

\author{
Morteza Mortazavi Mehrizi1 ${ }^{1}$ Mehrzad Ebrahemzadih ${ }^{2,3}{ }^{*}$, Abdolhamid Tajvar $^{4 *}$, \\ Omid Giahi' ${ }^{2,3}$ \\ ${ }^{1}$ Department of Occupational Health, School of Health, Shahid Sadoughi University of Medical Sciences, Yazd, \\ Iran \\ ${ }^{2}$ Kurdistan Environmental Health Research Center, Kurdistan University of Medical Sciences, Sanandaj, Iran \\ ${ }^{3}$ Department of Occupational Health, Faculty of Health, Kurdistan University of Medical Sciences, Sanandaj, \\ Iran \\ ${ }^{4}$ Research Center for Social Determinants of Health Promotion, School of Health, Hormozgan University of \\ Medical Sciences (HUMS), Bandar Abbas, Iran \\ Email: "Emhrzad@yahoo.com,
}

Received 19 October 2014; revised 6 December 2014; accepted 17 December 2014

Copyright (C) 2014 by authors and Scientific Research Publishing Inc.

This work is licensed under the Creative Commons Attribution International License (CC BY).

http://creativecommons.org/licenses/by/4.0/

c) (7) Open Access

\section{Abstract}

Aim: The first purpose is to survey the cumulative trauma disorder (CTD) prevalence in 4 zones of neck, shoulder, hand/wrist and back, and the second purpose is to survey the risk factors producing upper limb musculoskeletal disorders by using OCRA index method. In this analytic-descriptive and cross-sectional study, incorporation of four data collection methods was contained; observing and interviewing methods, using questionnaire and check list, were utilized. Randomly, 384 samples for CTD prevalence and 423 samples for OCRA index exposure survey were collected by cluster ratio sampling. In this study, findings indicated that CTD prevalence was high in studied bakery workers and most disorder and pain were reported in back, knees and hand/wrist. Through different tasks, most percentage of neck CTD (7.1\%) and shoulder CTD $(27.8 \%)$ was related to selling and baker tasks respectively, and most percentage of hand/wrist CTD (33/3\%) and back CTD (38.1\%) was related to bread baker task. There was a significant correlation between shoulder, hand/wrist and back CTD with bakery and task type, and effect of job background on CTD prevalence was improved in the above four zones. The rather high prevalence of CTD in the young population with average age of $\mathbf{3 0}$ years which have a few work experiences and the results of per-

\footnotetext{
${ }^{*}$ Corresponding authors.
} 
formed risk assessment can be taken account of serious warning, if it is not considered.

\title{
Keywords
}

\author{
OCRA Index, Musculoskeletal Disorders, Cumulative Trauma Disorder (CTD), Nordic \\ Questionnaire
}

\section{Introduction}

In the industrial world today, force labors, have to conform and fit to poor condition imposed by environment and applied tools, and accept existent limitation. The consequence of such compromise can be so serious and have an undesirable effect on individual life quality and health. In such situation man does not fit with work environment, type of work or equipment that is used, physically and mentally and continuance of this situation can result in musculoskeletal disorders which are the most common and main factors in losing work time, increasing costs and workforce injuries and according to research recently performed, they are taken to account cause of more than half of absenteeism in work environment and one third of repayments of work [1]-[3] and according to NIOSH categories, these disorders are in the second rank after pulmonary diseases in terms of prevalence, intensity and prevention possibility [1]. Various job risk factors can cause work-related musculoskeletal disorders whose important factors are related to physical work (such as posture, force, movement and vibration); psychosocial and individual factors [1] [4] [5]. According to national institute of occupational and safety health (NIOSH), cumulative trauma disorder (CTD) is one or more signs such as pain, creeping and rigidity, and movement restriction in one of four joint zones of upper limb (neck, shoulder, elbow, forearm and wrist) and lumbar last more than one week or repeat at least one time at month in last year providing there is not any acute and previous injuries for related joint [6]-[8]. There are three main characteristics for these disorders: a) this is accumulated in muscles over the time, b) the role of physical and mechanical stresses, c) persistence of disorder when getting out of normal condition [1] [3]. When in a job, repetitive work activities are more than worker's capacity and ability, defects create. As indicated, traumas from organ's repetitive movement contain cumulative characteristics that promote in time lapse and show the effects in long time as injuries involving musculoskeletal system. Hence repetitive exposures to producer factors of trauma result in cumulative injuries in muscles, tendons, ligaments, nerves, joints and blood vessels that sequentially cause inflammation, movement restriction and special disorders in these systems [1] [4]. One of the most important jobs which because of many repetitive movements, performing job in standing posture for a long time, wrong workstations and other factors such as psychological factors and improper environmental condition can cause work-related musculoskeletal disorders, is bakeries. So, prevention of these disorders outbreak needs workstations assessment using ergonomics job analysis methods. Nowadays, there are different methods for job exposure assessment to determine risk factors producing musculoskeletal disorders that in this study OCRA index method is used as most perfect methods to evaluate upper limb extremity exposure to repetitive movement [5] [6] [9].

\section{Methodology}

In this study, incorporation of four data collection method contains; observing and interviewing methods, using questionnaire and check list were utilized. All the participants in this study were men, we are selected randomly, 384 samples for CTD prevalence and 423 samples for OCRA index exposure survey collected by cluster ratio sampling. Kruskal-Wallis test, K square, repeated measures analysis of variance, Mann-Whitney test was performed by SPSS15 software for proving hypothesis.

Standard Nordic Musculoskeletal Questionnaire (NMQ) and National Institute of Occupational and Safety Health (NIOSH) new CTD definition was used to survey cumulative trauma disorder (CTD) prevalence. according to NIOSH new definition, CTD is one or more signs such as pain, creeping, rigidity and movement restriction in one of four joint zone of upper limb (neck, shoulder, elbow, forearm and wrist) and lumbar last more than one week or repeat at least one time at month in last year providing there is not any acute and previous injuries for related joint [7] [8]. Therefore after data collection, the subjects that contain above definition were extracted and categorized in four groups involve CTD in neck, shoulder, wrist/hand and lumbar, also Chi-square 
test was used to survey the effect of bakery type, task type and work background on CTD prevalence and for calculating exposure to repetitive job activities index, at first 423 samples randomly collected by cluster ratio sampling and whereas dispense ratio of four studied bakery type in Kerman, using video recording technique and after different job and task analysis and assigning the required risk factors quantities, by entering them to OCRA software. The OCRA index quantity and each of studied job and task risk level status was calculated and then was evaluated according to new category recently was developed by innovators of this method [9] (Table 1). It is noted that in order to obtain the average of OCRA index for different jobs, at first each of the jobs (bakeries) was separated to related tasks (contains bread baker, baker, seller agent, counter hand, counter hand/seller agent tasks) (Table 2) and required technical actions for performing the tasks and then doing an organizational analysis, repetitive tasks and the real duration of performing the tasks, number of cycles performed in repetitive tasks duration and cycle duration was determined and finally by determining main factors specifying job exposure involves repetitive movement, force exertion, movement and posture type, distribution of recovery times and other impressive factors (further factors) using following formula:

$$
\text { OCRA }=\mathrm{IE}=\frac{\text { Number of Activities Observed in Repetitive Tasks }}{\text { Number of Recommended Activities (Allowale) }}=\frac{\mathrm{Ac}}{\mathrm{Ar}}
$$

And by helping OCRA software, the exposure to repetitive job activities for right and left hand was calculated separately [7] [8] [10].

\section{Result}

After survey and analysis of demographic characteristics questionnaire, it was concluded that all the participants in this study were men and $65 \%$ of them were married and through of them only $14.1 \%$ have degree or higher. Also it was specified that most of subjects in study were young and had a few work background as only $17 \%$ of them were more than 40 years old and only $18.8 \%$ had 20 and more year work background. $88.8 \%$ of them was right-hand and $62.8 \%$ of them had $165-184 \mathrm{~cm}$ length and $60.4 \%$ of them was at the range of $61-80 \mathrm{~kg}$ weight.

Generally, through out of 384 bakery workers from ROTATORY and TRADITIONAL TAFTOUN, SANGAK and BAGUETTE participating in this study and perform bread baker, bakery, seller agent, counter hand, counter hand/seller agent tasks, 298 subjects were observed have CTD in neck, shoulder, wrist/hand, lumbar that through these 91 subjects were related to bread baker, 149 subjects were related to bakers, 10 subjects were related to bread makers, 6 subjects were related to sellers and 42 subjects were related to bread maker/sellers. According to participants ratio from each bakery and each task in this study conclude that through different tasks, most percentage of neck CTD (7.1\%), shoulder CTD (27.8\%) was related to, respectively, selling, baker tasks and most percentage of hand/wrist CTD (33/3\%) and back CTD (38.1\%) was related to bread baker task (Figure 1). Also between different bakeries most percentage of neck and lumbar CTD respectively with 7.7\% and 50\% was reported through SANGAK bakery workers and most percentage of shoulder and hand/wrist CTD respectively was reported through BAGUETTE bakery workers (Figure 2).

Also results of Chi-square test that was performed to survey the effect of bakery type and task type and work background on CTD prevalence in four zone of neck, shoulder, hand/wrist and lumbar, indicated that by $95 \%$ of confidence, bakery type and task type do not affect in creating neck CTD but affect in creating shoulder, hand/ wrist and lumbar CTD (Table 3). Also it was specified work background is effective factor in creating four considered zone CTD.

And about assessment results performing by OCRA technique as observed in Figure 3 and Figure 4, most OCRA index average of right and left hand was related to baking task in SANGAK bakery (OCRA index = 14.99) while least OCRA index average of right hand was related to selling task in SANGAK bakery (OCRA index $=0.69$ ) and least OCRA index average of left hand was calculated for selling task in ROTATORY TAFTOUN (OCRA index $=0.66)$.

Little attention in these graphs can find that OCRA index average of right and left hand in seller agent, counter hand, counter hand/seller agent tasks is the same however there is significant difference between OCRA index average of left hand in bread baker and baking tasks and OCRA index average of right hand in the same tasks that these results were also achieved by Mann-Whitney test with $95 \%$ confidence. 
Table 1. Final assessment criterion.

\begin{tabular}{cccl}
\hline OCRA Index Quantities & Zone & Risk Level & \multicolumn{1}{c}{ Required Measurement } \\
\hline 2.3 & Green & No risk & $\begin{array}{l}\text { Acceptable risk level and modified measurements is not } \\
\text { necessary. }\end{array}$ \\
$2.3-3.5$ & Yellow & Low risk & $\begin{array}{l}\text { It is recommended that according to structural risk factors } \\
\text { (posture, force, technical activities...) or by performing } \\
\text { organizational measurements, implements required modifications. }\end{array}$ \\
$>3.5$ & Red & Risky & $\begin{array}{l}\text { It is suggested that according to preferences for work environment } \\
\text { and considered task, redesign should be performed. }\end{array}$ \\
\hline
\end{tabular}

Table 2. Explanation of job titles in bakeries of Iran.

\begin{tabular}{cl}
\hline Job & \multicolumn{1}{c}{ Explanation } \\
\hline Bread baker & $\begin{array}{l}\text { Performing works like formation and processing of dough; bread rolling; unloading flour sacks; adding } \\
\text { necessary enzymes to the dough in mixer; and tools' dusting by flour }\end{array}$ \\
Baker & Bread's baking and finishing \\
Counter hand & Assistant of confectioner \\
Seller agent & Collecting baked bread and selling to the customer \\
Counter hand/seller agent & Multi functional job
\end{tabular}

Table 3. Relation between bakery type, task type and work background with CTD prevalence that was measured by Chisquare test in significant level of 0.05 .

\begin{tabular}{cccc}
\hline CTD type & Bakery type & Task type & Work background \\
CTD in neck & 0.432 & 0.209 & 0.002 \\
CTD in shoulder & 0.006 & $<0.0001$ & $<0.0001$ \\
CTD in hand/wrist & 0.039 & $<0.0001$ & $<0.0001$ \\
CTD in lumbar & 0.018 & $<0.0001$ & $<0.0001$ \\
\hline
\end{tabular}

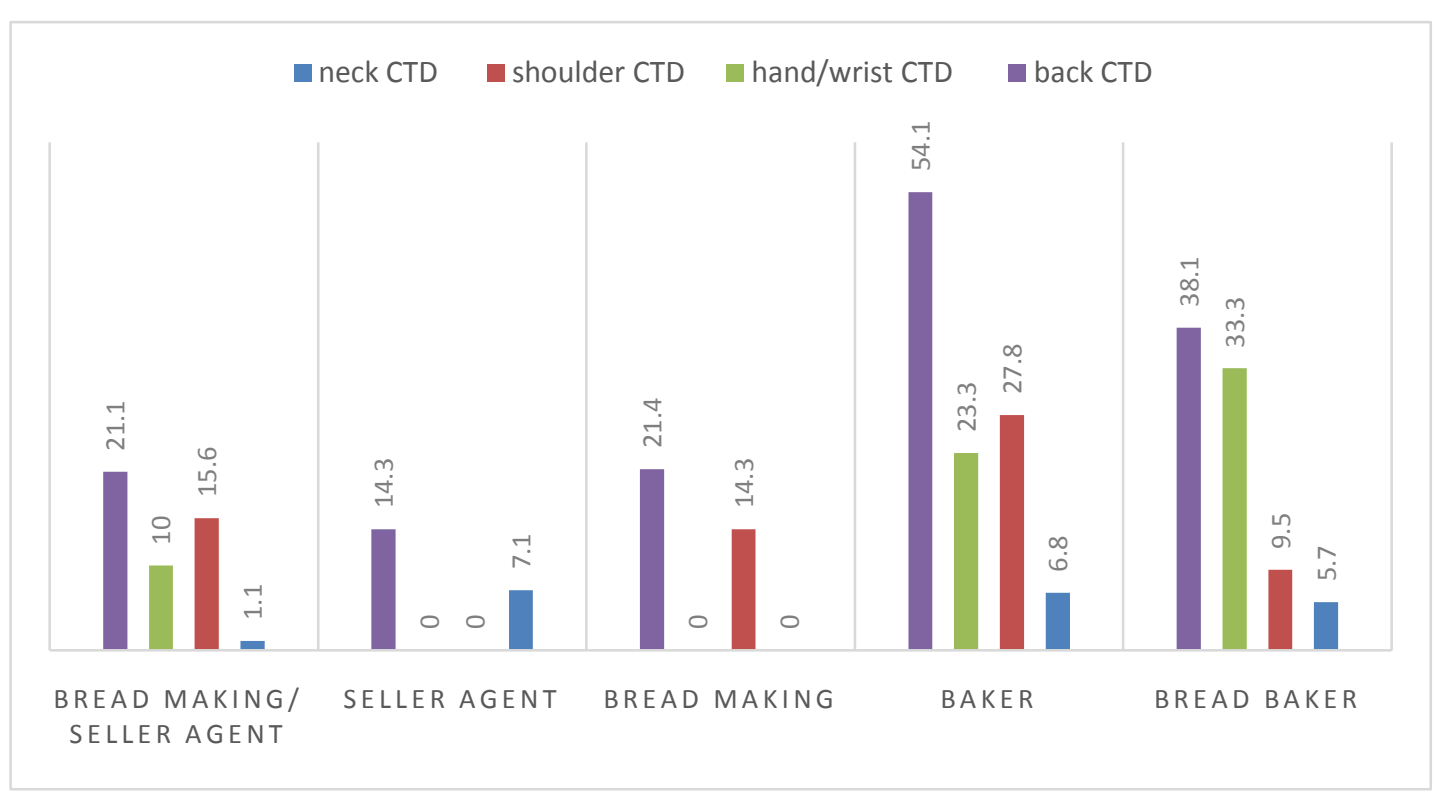

Figure 1. Most percentage of neck, shoulder, hand/wrist and lumbar CTD through different tasks. 


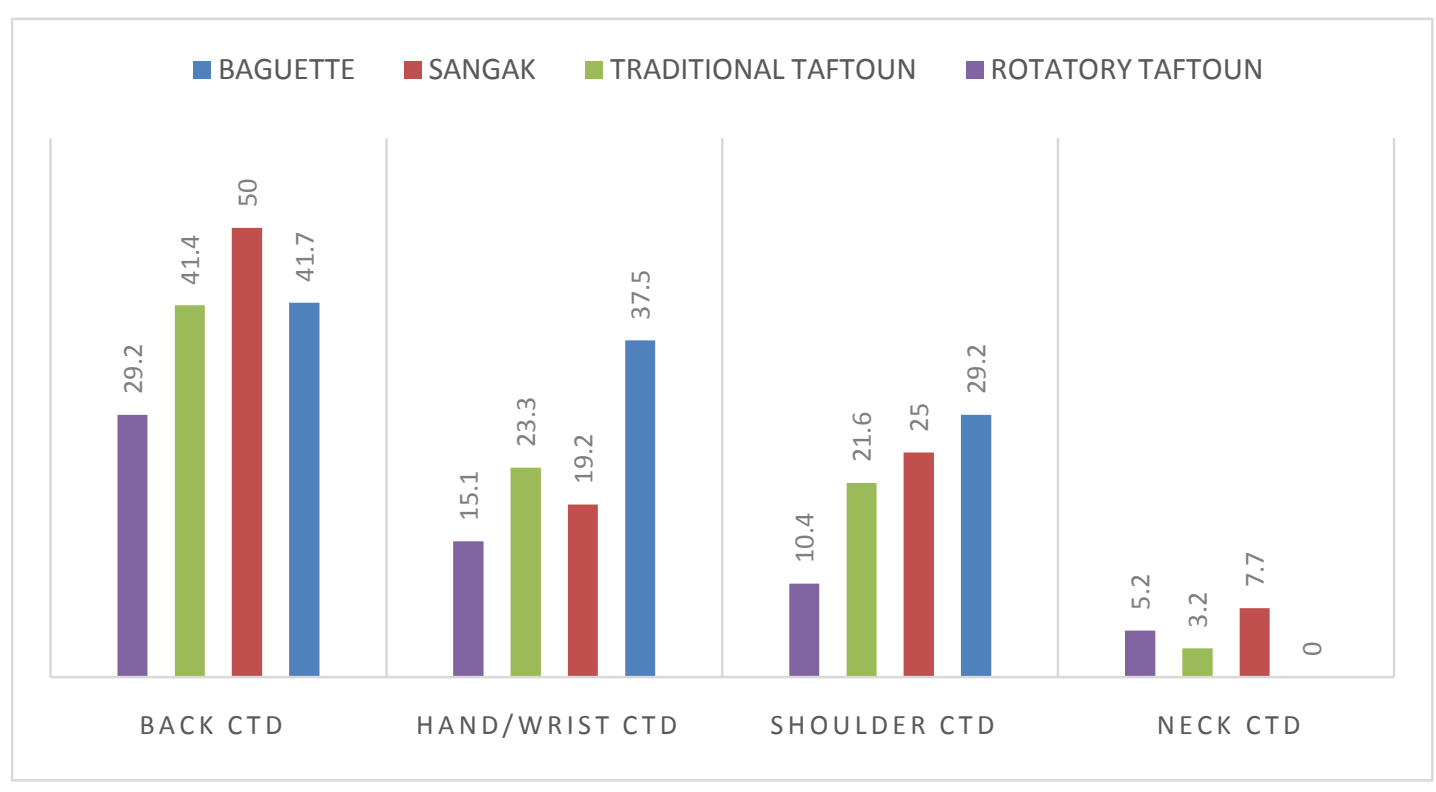

Figure 2. Most percentage of neck, shoulder, hand/wrist and lumbar CTD through different bakeries.

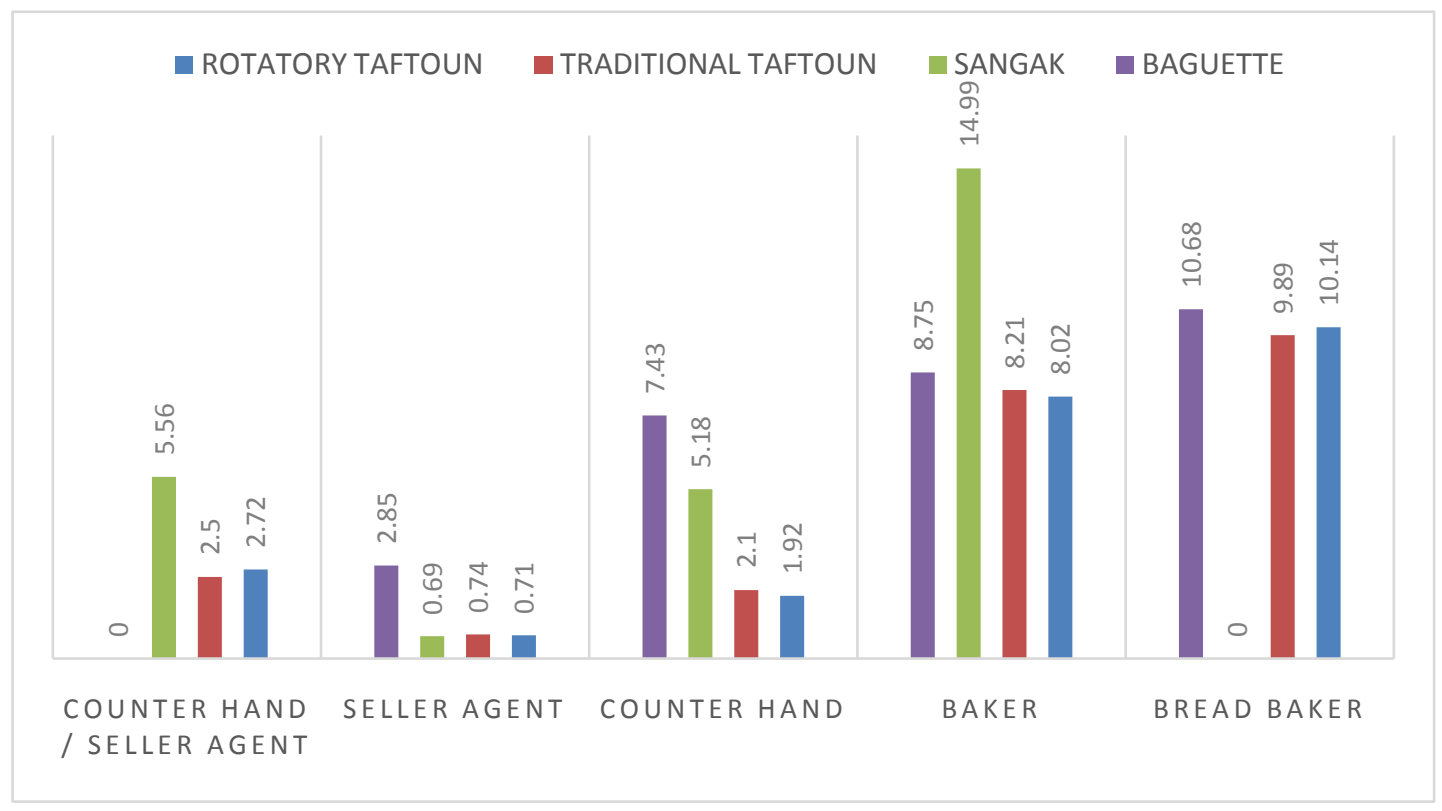

Figure 3. Most OCRA index average of right hand for different tasks in studied bakeries.

\section{Conclusions}

The rather high prevalence of CTD in studied population that was young (with age average of 30 years) and had a few job background and results of performed risk assessment can be taken account of serious warning and if it is not considered, it will probably impose enormous costs on government and society in future.

On the other hand, after determining risk levels for right and left hand of each task in bakeries, it was specified that risk level of bread baker and baking tasks in every four bakeries as well as counter hand task in SANGAK and BAGUETTE bakeries and counter hand/seller agent in SANGAK bakery were in red zone or danger zone. So redesign for modifying work condition is necessary. With regard to the fact that $5.6 \%$ of total tasks in ROTATORY TAFTOUN bakery, $67.4 \%$ in TRADITIONAL TAFTOUN bakery, $77.3 \%$ in SANGAK bakery and $75 \%$ in BAGUETTE bakery were in red or danger zone, therefore it can be concluded that most 


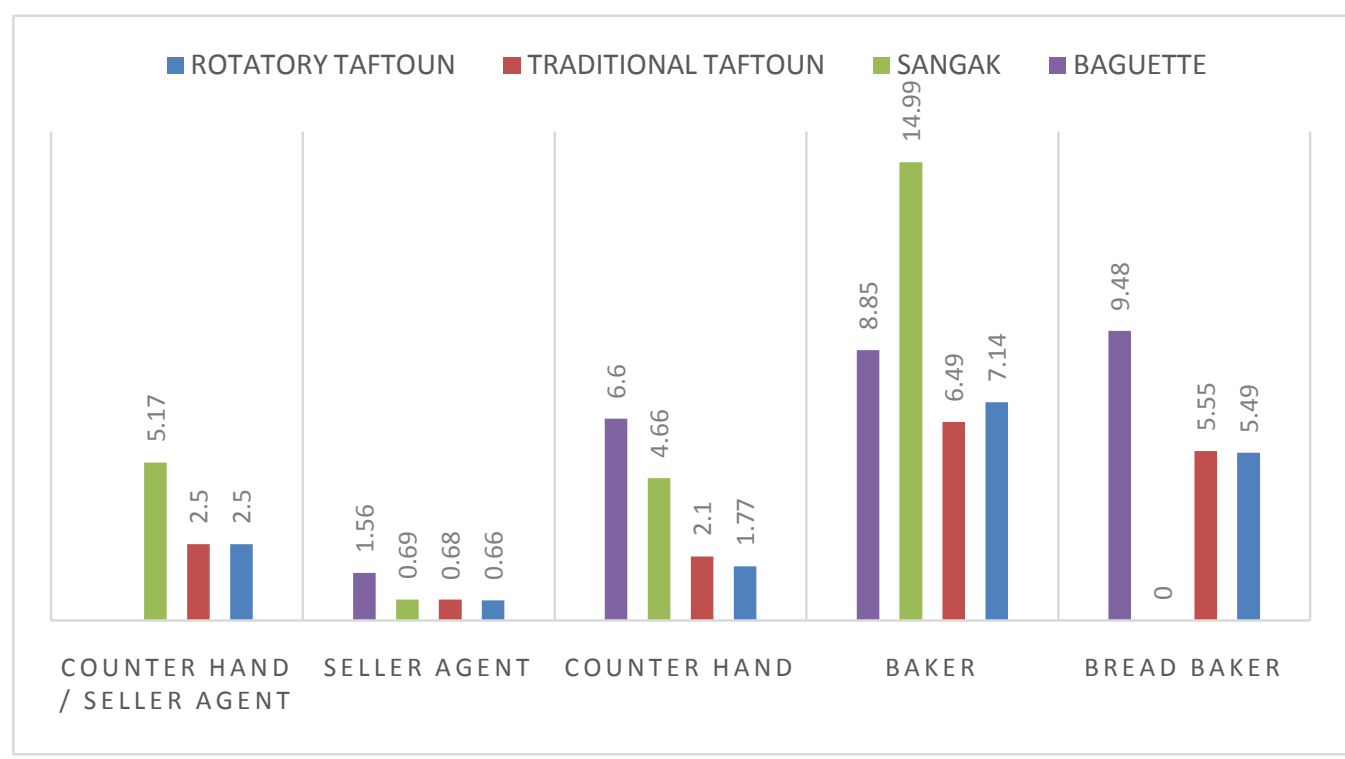

Figure 4. Most OCRA index average of left hand for different tasks in studied bakeries.

working bakeries in Kerman-Iran are at risk for musculoskeletal disorders and thus if it is not considered, it will probably impose enormous costs on government and society in future. So we should think about solutions that by executing them can modify this condition and certainly the best suggestion is the ergonomic control methods as most important part in every ergonomic program and the effect of them in reducing work related musculoskeletal disorders was proved till now [11] [12]. Redesign in whole bread baking systemic recommended as the first and best remedy for reducing and even eliminating musculoskeletal disorders (as well as OCRA software was recommended too). On the other hand, it is better to say, best remedy is replacing the mechanical bread instead of traditional bread. Actually by implementing this we can eliminate the process of musculoskeletal disorders creation. This conclusion was confirmed after video tape recording from mechanical bread baking method and calculating the OCRA index for it (OCRA index $=1)$.

\section{Discussion and Suggestion}

Executing this plan has so many economical utilities for government; however there is a big problem in front of this plan that is the food taste in bread selection in Iranian culture especially in Kerman that most of people choose the traditional bread and do not have a good relationship with mechanical bread. The evidence for this claim is that most of mechanical bread baking experimental plans executing in some area in Iran, do not meet with general acceptance till now. Thus we have to now abandon this plan with all its utilities and think about using other control methods that can point to following instances:

We should consider suitable work table according to bakers' job demands and anthropometry, using anti-fatigue flooring, electronic baker device, proper and ergonomic tools like using ergonomic spatula while cutting paste in BAGUETTE bakery and education to bakers about musculoskeletal disorders and the way of prevention of them besides using management methods such as work rotation or short time rest.

Since many studies shows that using combination of these methods follows better results [13], we also propose that the possible combination of these methods is used to modify work environment in bakery job. Recognized ergonomic injuries are inherently personal in nature and contributed to by ongoing perceptions, decisions, and methods, and ergo-related soft tissue and other injuries can be directly affected by off-work activities. So, sometimes out of frustration with having abortively tried other approaches, this culture focuses on self-directed ergonomics from inside to outside, with each worker becoming his or her own "ergonomic expert" [14].

\section{References}

[1] Marras, W.S. and Karwowski, W. (2010) Fundamentals and Assessment Tools for Occupational Ergonomics. CRC Press, Boca Raton. 
[2] Molteni, G., De Vito, G., Sias, N. and Grieco, A. (1996) Epidemiology of Musculoskeletal Disorders Caused Biomechanical Overload (WMSDs). La Medicina del Lavoro, 87, 469-481.

[3] Rizzo, T.H., et al. (1997) Reducing Risk Factors for Cumulative Trauma Disorders (CTDs): The Impact of Preventive ergonomic Training on Knowledge, Intentions, and Practices Related to Computer Use. American Journal of Health Promotion, 11, 250-253. http://dx.doi.org/10.4278/0890-1171-11.4.250

[4] Stobbe, T.J. (1995) Occupational Ergonomics and Injury Prevention. Occupational Medicine (Philadelphia, Pa.), 11, 531-543.

[5] Occhipinti, E. and Colombini, D. (2001) The OCRA Method: Assessment of Exposure to Occupational Repetitive Actions of Upper Limbs. In: Krawowski, W., Ed., International Encyclopedia of Ergonomics and Human Factors, Volume One, Taylor \& Francis, London \& New York, Impression 12, 1875-1879.

[6] Occhipinti, E. and Colombini, D. (1996) Proposal of a Concise Index for the Evaluation of the Exposure to Repetitive Movements of the Upper Extremity (OCRA Index). La Medicina del Lavoro, 87, 526-548.

[7] Armstrong, T., Buckle, P., Fine, L., et al. (1993) A Conceptual Model for Work-Related Neck and Upper Limb Musculoskeletal Disorders. Scandinavian Journal of Work, Environment \& Health, 19, 73-84. http://dx.doi.org/10.5271/sjweh.1494

[8] Keyserling, M., Brouwer, M. and Silverstein, B. (1992) A Checklist Forevaluating Ergonomic Risk Factors Resulting from Awkward Postures of the Legs, Trunk, and Neck. International Journal of Industrial Ergonomics, 9, $283-301$. http://dx.doi.org/10.1016/0169-8141(92)90062-5

[9] Colombini, D. and Occhipinti, E. (2006) Preventing Upper Limb Work-Related Musculoskeleyal Disorders (ULWMSDS): New Approaches in Job (Re)design and Current Trends in Standardization. Applied Ergonomics, 37, 441-450. http://dx.doi.org/10.1016/j.apergo.2006.04.008

[10] Moussavi-Najarkola, S.A. (2006) Concise Exposure and Damage Indicator for Predicting Foreseeable Effects of WorkRelated Upper Limb Disorders. Iranian Journal of Public Health, 35, 75-83.

[11] Ayoub, M.A. (1990) Ergonomic Deficiencies: II. Probable Causes. Journal of Occupational and Environmental Medicine, 32, 131-136.

[12] Tayyari, F. and Smith, J.L. (1997) Occupational Ergonomics: Principle and Applications. Chapman and Hall, 11, 5459.

[13] Silverstein, B. and Clark, R. (2004) Intervention to Reduce Work-Related Musculoskeletal Disorders. Journal of Electromyography and Kinesiology, 14, 135-152. http://dx.doi.org/10.1016/j.jelekin.2003.09.023

[14] Halvani, G., Ebrahimzadeh, M., Dehghan, M., Fallah, H. and Mortazavi, M. (2012) Assessment of Factors Affecting Safety Culture in Yazd Steel Industry Workers. Occupational Medicine Journal, 4, 66-72. 
Scientific Research Publishing (SCIRP) is one of the largest Open Access journal publishers. It is currently publishing more than 200 open access, online, peer-reviewed journals covering a wide range of academic disciplines. SCIRP serves the worldwide academic communities and contributes to the progress and application of science with its publication.

Other selected journals from SCIRP are listed as below. Submit your manuscript to us via either submit@scirp.org or Online Submission Portal.
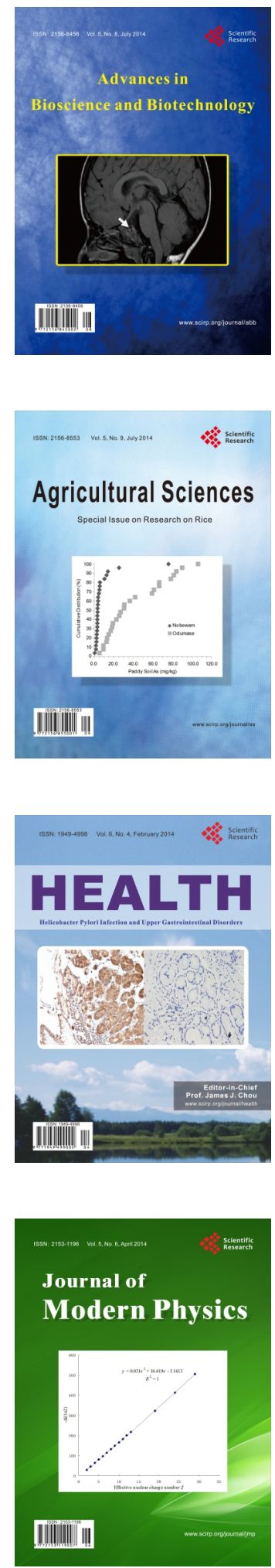
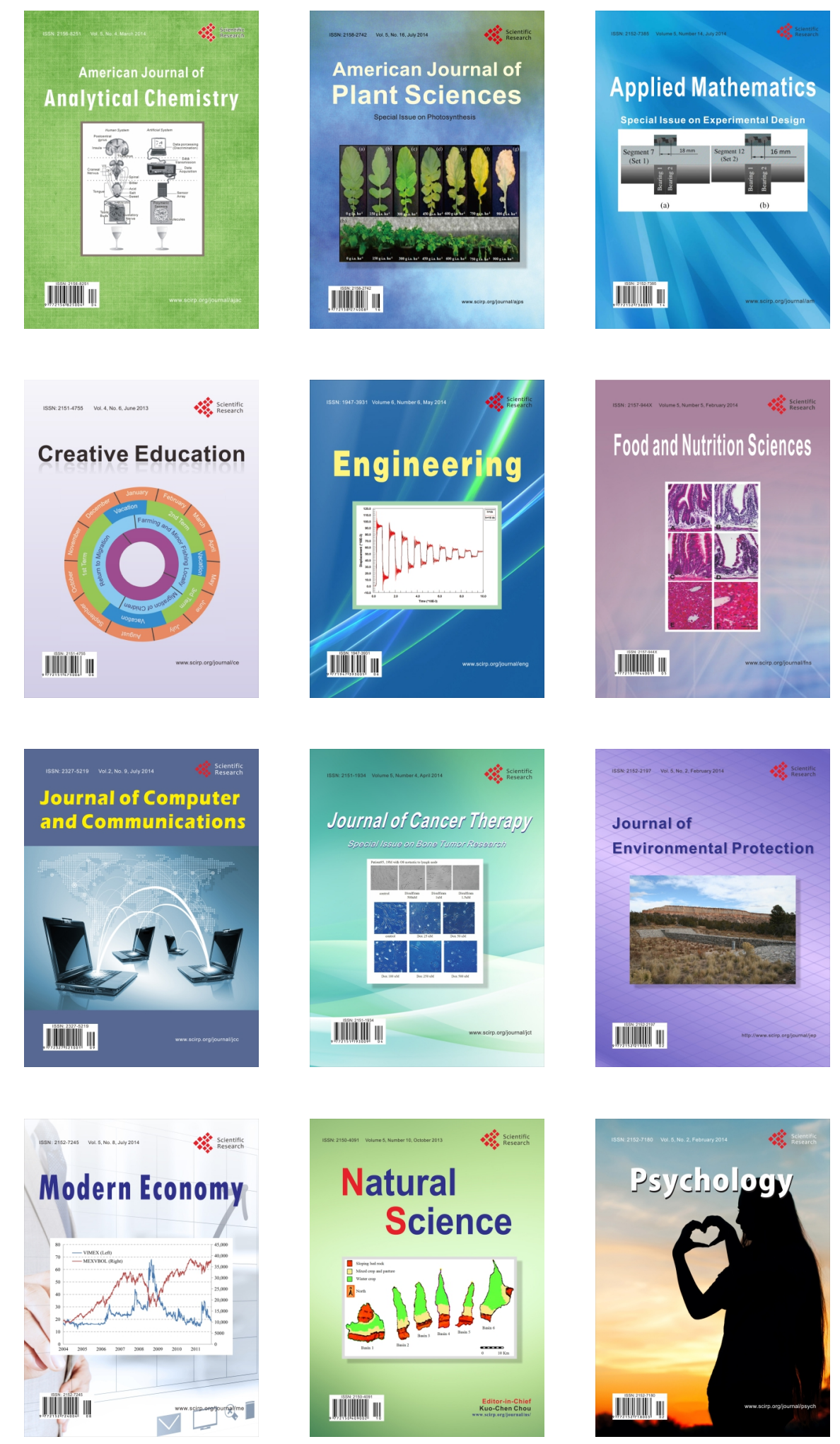